\title{
Frequency-Domain Multiplexed Readout for Superconducting Gamma-Ray Detectors
}

\author{
Jonathan G. Dreyer, Kam Arnold, Trevor M. Lanting, Matt A. Dobbs, Stephan Friedrich, Adrian T. \\ Lee, and Helmuth G. Spieler
}

\begin{abstract}
We are developing a frequency-multiplexed readout for arrays of high-resolution Gamma detectors based on superconducting transition edge sensors (TESs). Each sensor is part of an LCR resonant circuit and is biased at an identifying carrier frequency. Several carrier signals are added and amplified with a single SQUID preamplifier at $4 \mathrm{~K}$. Gamma absorption modulates the amplitude of the carrier, and demodulation at room temperature retrieves the initial temperature evolution of the sensor. This multiplexing system has originally been developed to read out large arrays of bolometers for cosmic microwave background studies. To accommodate the faster Gamma-ray signals, its demodulator bandwidth is being extended to $\sim 20 \mathrm{kHz}$ to allow reading out up to eight TESs with a detector bandwidth of $\sim 10 \mathrm{kHz}$. Here we characterize the system noise performance and show how this multiplexing scheme can be adapted to read out arrays of superconducting Gamma-ray detectors.
\end{abstract}

Index Terms - Gamma ray detectors, frequency domain multiplexing, superconducting radiation detectors, transition edge sensors.

\section{INTRODUCTION}

G AMMA ray spectrometers based on superconducting $\mathbf{J}_{\text {transition edge sensors offer an order of magnitude higher }}$ energy resolution than conventional detectors based on highpurity germanium crystals [1]. This can improve the precision of non-destructive isotope analysis by gamma spectroscopy in nuclear science and national security applications [2]. Over the last decade, the energy resolution of TES Gamma-ray

This work was supported by the U.S. Department of Energy, Office of Nonproliferation Research and Engineering NA-22 under grant LL035-DP, and by the NASA APRA program under grant NNG04WF071. Part of this work was performed under the auspices of the U.S. Department of Energy by University of California Lawrence Livermore National Laboratory under contract No. W-7405-Eng-48.

J. G. Dreyer and S. Friedrich are with the Advanced Detector Group at the Lawrence Livermore National Laboratory, Livermore, CA 94550, USA (email: friedrich1@1lnl.gov; phone 925-423-1527; fax: 925-424-5512). J. D. Dreyer is also with the Department of Nuclear Engineering at the University of California, Berkeley, CA 94720, USA.

T. M. Lanting, Kam Arnold, and A. T. Lee are with the Physics Department at the University of California, Berkeley, CA 94720, USA (email: at1@physics.berkeley.edu).

Matt A. Dobbs was with the Physics Division at the Lawrence Berkeley National Laboratory and is now at McGill University in Montreal, Canada. H. G. Spieler is with the Physics Division at the Lawrence Berkeley National Laboratory, Berkeley, CA 94720, USA (e-mail: HGSpieler@lbl.gov), supported by the DOE Office of Science, Office of High Energy and Nuclear Physics, under Contract No. DE-AC02-05CH11231. detectors has been improved to well below $100 \mathrm{eV}$ FWHM at $100 \mathrm{keV}[3,4]$, which is sufficient for most nuclear analyses $[2,3]$. But since the volume of individual sensors is limited to $\sim \mathrm{mm}^{3}$ and the $\sim \mathrm{ms}$ decay time constants prevent data acquisition at high rates, we are developing pixilated detector arrays for increased sensitivity. The photolithographic fabrication of detector arrays is comparably straightforward, but the readout of multiple pixels in the arrays is challenging because of the thermal load associated with a larger number of wires to the detector cold stage. Operating large arrays therefore requires the development of a multiplexing scheme to read out large arrays with a small number of wires to reduce the associated heat load. Such a design will dramatically increase detector efficiency without significantly affecting the hold time of the cryostat.

Several multiplexing methods have been put forth for cosmic microwave background and x-ray observations. The usage of time-domain multiplexing, has been demonstrated for 32 sensors [5]. More recently frequency-domain multiplexing has been demonstrated using a single amplifier with up to 8 TES channels [6, 7].

We are developing frequency division multiplexers because their power dissipation at low-temperature is less and the low temperature circuitry is significantly simpler than for time division multiplexers. Here we discuss the adaptation of a frequency-domain multiplexed readout developed for TES microwave bolometers for use with TES Gamma-ray arrays. Although the operating principle for the readout will remain unchanged, we address the modifications necessary to optimize the performance of the system, thus maintaining the ultra high resolution required for non-destructive isotope analysis.

\section{SYSTEM DESCRIPTION}

Each TES with resistance $\mathrm{R}_{\mathrm{TES}}$ is biased in an $\mathrm{LC}_{\mathrm{i}} \mathrm{R}_{\mathrm{TES}}$ resonance circuit with a center frequency $\omega_{i}=\left(L C_{i}\right)^{-1 / 2}$. The bandwidth $b=R_{T E S} / L$ selected for each tuned circuit is set by the thermal time constant of the sensor. The spacing between the individual bias frequencies is chosen to minimize crosstalk.

Bias currents from multiple carrier sources are summed in a low-value bias resistor $R_{\text {bias }}$ to form the low-impedance voltage source needed to ensure constant voltage bias operation. The bias frequencies range from 300 to $700 \mathrm{kHz}$. 


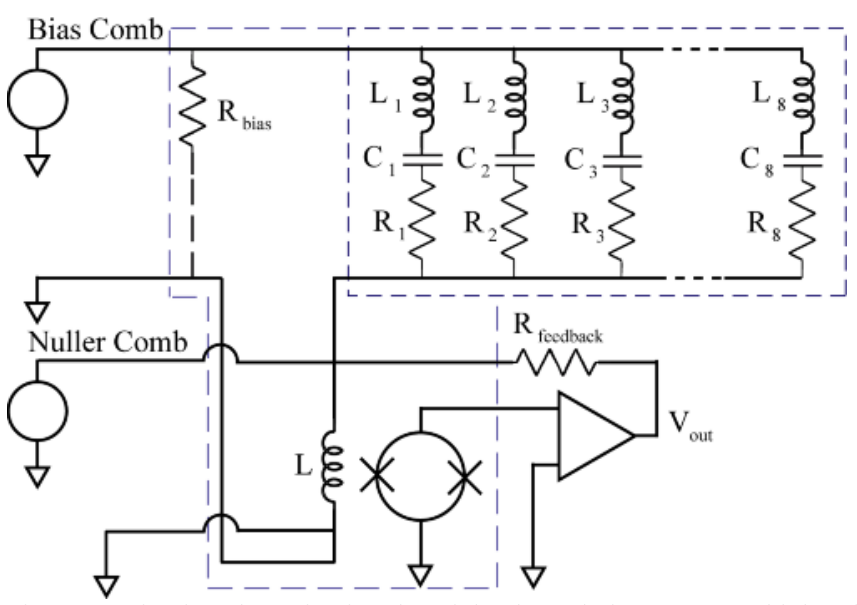

Fig. 1. Circuit schematic for the eight-channel frequency multiplexed readout. For system characterization, calibrated $0.46 \Omega$ resistors were used for all eight channels.

The amplitude of the carrier associated with each detector is modulated by changes in TES resistance. Since each detector is biased at a different frequency, the individual detector signals are uniquely associated with discrete bands in frequency space. These individual signals are then summed into a single SQUID preamplifier, preserving the amplitude modulation of each carrier. To reduce the dynamic range requirements for the SQUID, a nulling comb that is $180^{\circ}$ out of phase with the bias carriers is applied to the SQUID. This suppresses the bias carrier while the modulated sensor signal is preserved for readout. Shunt feedback through $R_{\text {feedback }}$ establishes a low input impedance at the SQUID input, The output of the SQUID amplifier fed to a bank of demodulators, one for each bolometer, that translate the individual bolometer signals into original signal band. Onboard ADCs sample the output signals and the digitized signals are sent to a computer for subsequent signal processing.

\section{IMPLEMENTATION}

Implementation of frequency-domain multiplexing for highresolution Gamma detectors requires the modification of several components in the existing readout system originally designed for microwave bolometers. The major change is due to the higher sampling frequency required for the faster sensors used here. This requires changes to the firmware that controls the readout logic and the bandwidth of the postdetection anti-aliasing filter must be increased from $400 \mathrm{~Hz}$ to $20 \mathrm{kHz}$ to match the higher sampling rate. The parameters of the sensors are also chosen to facilitate the required changes. These components are inexpensive and can be modified without significantly changing existing electronics.

The component values of the LC filter are set by the requirements of the readout electronics and TES detectors. These values must be optimized to offer sufficient signal bandwidth and limit cross talk between channels, while still remaining within the maximum frequency range of $1 \mathrm{MHz}$ for multiplexing system. To achieve constant bandwidth each multiplexer branch operates with the same inductance and the capacitance is chosen to set the individual resonant frequencies. We have initially chosen inductances of $L=$ $16 \mu \mathrm{H}$, which are available as chips that have already been well-characterized and proven to work well in multiplexing systems. The capacitance values are selected according to $C_{\mathrm{i}}=1 /\left(4 \pi^{2} L f_{\mathrm{i}}^{2}\right)$ where $f_{\mathrm{i}}$ is the center frequency of channel $\mathrm{i}$ and $L$ is the inductance of $16 \mu \mathrm{H}$. For a spacing of $\sim 50 \mathrm{kHz}$ between bias frequencies, the capacitor values range from 4 to $20 \mathrm{nF}$. NPO chip capacitors are used, whose temperature dependence has been characterized to $4.2 \mathrm{~K}$. The capacitance linearly decreases to $85 \%$ of the room temperature value.

Electrothermal stability requires the LC filter bandwidth to be at least a factor six larger than the associated TES bandwidth, so for a detector time constant of $1 \mathrm{~ms}$, the bandwidth must be greater than $6 \times 160 \mathrm{~Hz}$. Furthermore, a signal bandwidth of $\sim 10 \mathrm{kHz}$ is required to read out the TESs with high signal-to-noise ratio (Fig. 3). So the bandwidth of each tuned circuit must be increased to accommodate twice the signal bandwidth, as the signal is mirrored in two sidebands above and below the carrier. For an $\mathrm{L}$ of $16 \mu \mathrm{H}$, the total resistance (TES and parasitic resistances) in series with the LC circuit is required to be of the order $1 \Omega$. Smaller inductors would allow operating TESs with lower resistances.

The Gamma-ray sensors used in the array are $\mathrm{Mo} / \mathrm{Cu}$ multilayers on thin $\mathrm{SiN}$ membranes that provide the weak thermal link to the cold bath. A bulk Sn absorber attached to each sensor increases the detection efficiency of the individual pixels $[2,3]$. A crucial feature of multilayer TESs is the ability to select the resistance of the sensor independently of the transition temperature (Fig 2). This characteristic allows for optimizing the resistance required for the readout electronics while still maintaining the desired transition temperature of $\sim 120 \mathrm{mK}$.

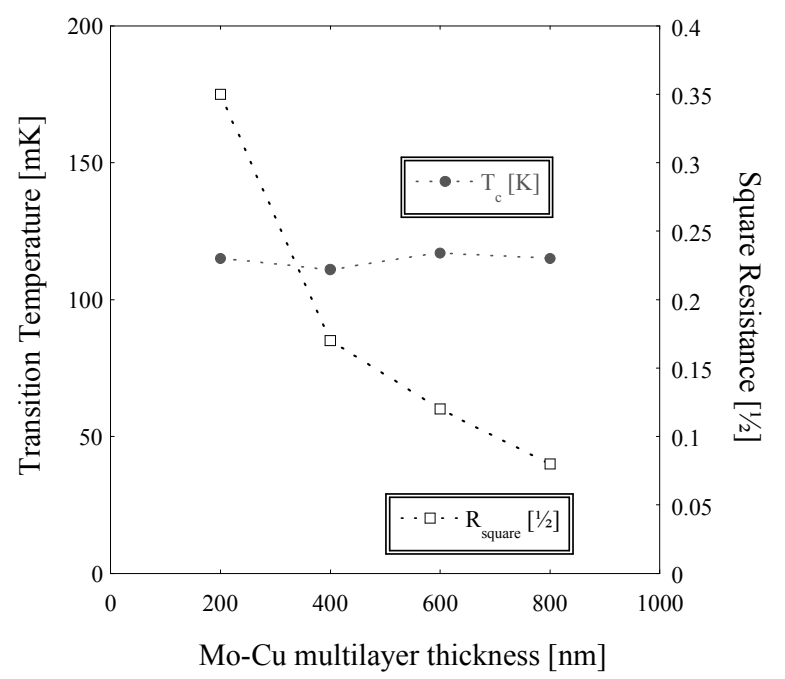

Fig. 2. Critical temperature and square resistance of $\mathrm{Mo} / \mathrm{Cu}$ multilayer TESs with a $\mathrm{Mo} / \mathrm{Cu}$ ratio of 4:9 for different multilayer thicknesses. Note that the multilayer approach allows adjusting the sensor resistance while keeping the transition temperature constant. 


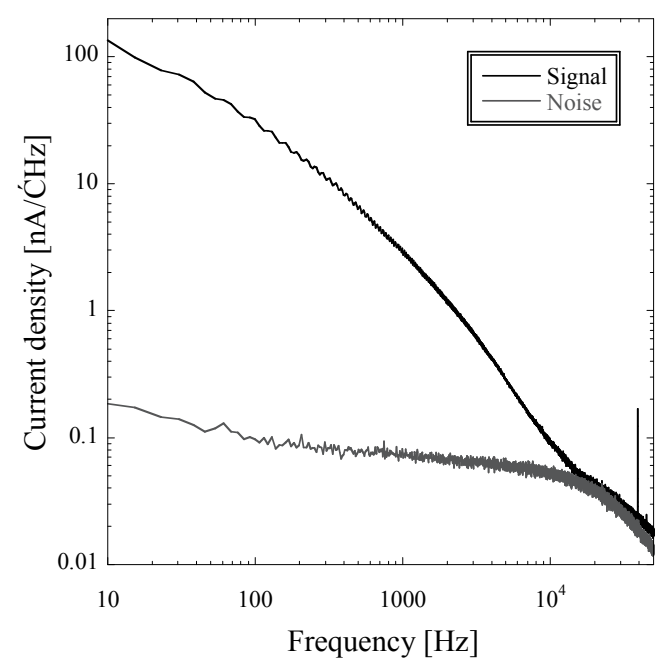

Fig. 3. Signal and noise frequency characteristics of typical composite TES Gamma-ray detectors. The white noise level is set by the phonon noise associated with the thermal conductance between the bulk absorber and the TES thermistor.

Multilayers with a thickness between 200 and $800 \mathrm{~nm}$ have resistances between 0.35 and $0.08 \Omega$, and we can increase the resistance into the $1 \Omega$ range with a multilayer thickness of $\sim 50 \mathrm{~nm}$.

To first order the phonon noise current in TES Gamma-ray detectors is given by

$$
i_{n} \approx \sqrt{4 k_{B} T^{2} G} \cdot \frac{1}{V_{\text {bias }}},
$$

where $T$ is the transition temperature of the sensors of $120 \mathrm{mK}$ $G$ is the thermal conductance between absorber and TES, approximately $10 \mathrm{nW} / \mathrm{K}$ depending on design, and $1 / V_{\text {bias }}$ is the responsivity, the conversion of phonon noise power into the input noise current. For $V_{\text {bias }} \approx 1 \mu \mathrm{V}$ we expect a sensor current noise on the order of $\sim 100 \mathrm{pA} / \sqrt{\mathrm{Hz}}$ (Fig. 3).

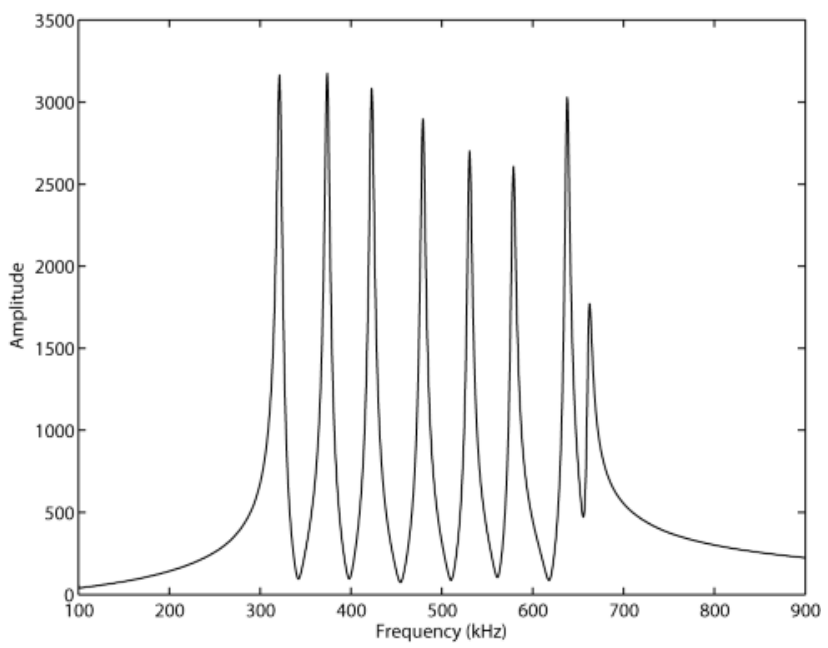

Fig. 4. Frequency response of the 8 channel multiplexing readout with 0.46 $\Omega$ resistors.

\section{SYSTEM CHARACTERIZATION}

To confirm that this frequency-domain multiplexing system can be used to read out large $\mathrm{Mo} / \mathrm{Cu}$ Gamma-ray TES arrays, a setup consisting of eight $0.46 \Omega$ resistors and LC filters with $16 \mu \mathrm{H}$ inductors and capacitors ranging from $\sim 5$ to $18 \mathrm{nF}$ at $300 \mathrm{~K}$ was assembled ( $c f$. Fig 1). The setup was placed in a test cryostat and cooled to a temperature of $1.8 \mathrm{~K}$. A variable frequency bias was applied from 100 to $900 \mathrm{kHz}$, and the output response was recorded (Fig. 4). The measured center frequencies and the widths of the eight peaks correspond to the expected LC resonances to within one percent, taking into account the reduction of the capacitance by $\sim 15 \%$ on cooldown. The response can be fine-tuned in future setups depending on the exact value of the TES resistance at the operating point.

The current noise of the multiplexer system was measured with the test resistors at $2.1 \mathrm{~K}$. This noise level was calculated by measuring the spectrum of the output of the SQUID electronics with an HP4195 network analyzer, and referring it to the SQUID input coil as a current noise (Fig 5). The total noise between the filter resonances is measured to be $\sim 5$ $\mathrm{pA} / \sqrt{\mathrm{Hz}}$, as expected from the SQUID characteristics and the Johnson noise of the feedback resistor. On resonance, a single $0.46 \Omega$ test resistor adds $\left(4 k_{B} T / R\right)^{1 / 2} \approx 16 \mathrm{pA} / \sqrt{\mathrm{Hz}}$ of noise. The measured average current noise on resonance of $\sim 18$ $\mathrm{pA} / \sqrt{\mathrm{Hz}}$ is consistent with the quadrature sum of the resistor's Johnson noise and the readout noise.

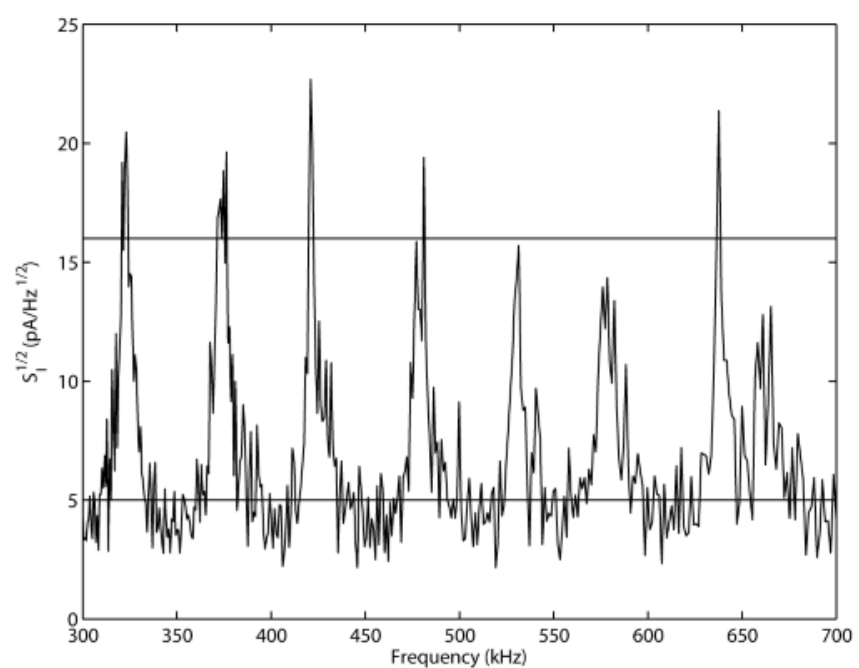

Fig. 5. Current noise from an eight-channel multiplexer, referred to the SQUID array input. At the resonance frequencies the noise increases due to the $0.46 \Omega$ resistor in the resonant circuit.

This noise floor of $5 \mathrm{pA} / \sqrt{\mathrm{Hz}}$ is more than adequate for TES gamma-ray sensors with a detector noise in the $\sim 50$ to 100 $\mathrm{pA} / \sqrt{\mathrm{Hz}}$ range. Future decreases of the inductance of the bandpass filter and fabrication of $\sim 1 \Omega$ TESs will ensure that the detector bandwidth will cover the full range of frequencies where the signal-to-noise ratio exceeds unity in our gamma detectors. In addition, we are currently changing the firmware 
to increase the sampling rate of the $\mathrm{A} / \mathrm{D}$ converter and the demultiplexer to $50 \mathrm{kHz}$, and are adjusting the Nyquist filters accordingly.

\section{SUMMARY}

We have characterized the response of our frequencymultiplexed readout for superconducting transition edge sensors. The response agrees with the design values, and the readout noise floor of $5 \mathrm{pA} / \sqrt{\mathrm{Hz}}$ is well below the intrinsic noise level of typical TES Gamma-ray detectors. We also show that this frequency domain multiplexing scheme can be adapted for the readout of arrays of TES gamma ray spectrometers with moderate modifications. Such arrays will provide increased detection efficiency without the significant heat load associated with arrays of non-multiplexed sensors. Therefore, this development will increase the sensitivity of TES Gamma-ray spectrometers for nuclear analysis, and of imaging spectrometers for high-energy astrophysics.

\section{REFERENCES}

[1] For an overview of the current status of cryogenic detector development, see, Nucl. Inst. Meth. A, vol. 559 (2006)

[2] O. B. Drury, S. F. Terracol, S. Friedrich, Quantifying the benefits of ultrahigh energy resolution for Gamma-ray spectrometry", Phys. Stat. Solidi C5, 1468-1479 (2005)

[3] S. F. Terracol et al., "Ultra-High Resolution Gamma-Ray Spectrometer Development for Nuclear Attribution and Non-Proliferation Applications", Conference Record, 2004 IEEE Nuclear Science Symposium, Rome (2004)

[4] J. N Ullom et al., Conference Record, 2005 IEEE Nuclear Science Symposium, Puerto Rico (2005)

[5] P. A. J. de Korte, J. Beyer, S. Deiker, G. C. Hilton, K. D. Irwin, M. Macintosh, S. W. Nam, C. D. Reinstsema, L. R. Vale, and M. E. Huber, Rev. Sci. Instrum. 74, 3807, 2003.

[6] Lanting et al. "Frequency-domain multiplexed readout of transitionedge sensor arrays with a superconducting quantum interference device" Appl. Phys. Lett. 86, 112511, 2005.

[7] Cunningham et al. "High-resolution operation of frequency-multiplexed transition-edge photon sensors." Appl. Phys. Lett., Vol. 81, 2002. 